\title{
Enhanced Phase Contrast Transfer using Ptychography Combined with a Pre- Specimen Phase Plate in a Scanning Transmission Electron Microscope
}

\author{
Hao Yang ${ }^{1}$, Colin Ophus ${ }^{1}$, Peter Ercius ${ }^{1}$ and Peter D. Nellist ${ }^{2}$ \\ 1. National Center for Electron Microscopy, Molecular Foundry, Lawrence Berkeley National \\ Laboratory, Berkeley, CA, 94720, USA. \\ 2. Department of Materials, University of Oxford, Parks Rd, Oxford UK.
}

The ability to image light elements in both crystalline and noncrystalline materials at near atomic resolution with an enhanced contrast is highly advantageous to understand the structure and properties of a wide range of beam sensitive biological specimens and molecular hetero-structures. This requires the imaging system to have an efficient phase contrast transfer function (PCTF) at both low and high spatial frequencies. Phase contrast imaging is generally performed in the conventional transmission electron microscope (CTEM), however, the PCTF of CTEM is not efficient especially at low spatial frequencies, and a large defocus has to be used to gain a necessary contrast of a weakly scattering specimen. The introduction of a post-specimen phase plate in TEM is found to enhance the low spatial frequency contrast transfer for example in [1], and there continues to be considerable interests in the development of such physical phase plates.

Recent development in high speed pixelated detectors has created opportunities for highly efficient phase imaging in a scanning transmission electron microscope (STEM). It allows the acquisition of two dimensional diffraction patterns at every probe position of a two dimensional electron beam raster across the specimen, forming an information-rich four dimensional (4D-) STEM dataset from which phase information can be extracted. For example, the atomic electric field within a thin specimen can be mapped out [2]. Ptychography 4D-STEM has also been shown to form an efficient and quantitative phase image under zero lens aberrations simultaneously with the incoherent Z-contrast imaging [3], and it has been shown to offer a relatively simple monotonic PCTF [4]. It has been shown recently that the introduction of a phase plate at the probe forming aperture of a STEM is equivalent to a Zernike phase plate in the back focal plane of a TEM [5], and the phase plate leads to a PCTF that depends on the cosine of the aberration wave shift [6]. Another study demonstrated that combining a pre-specimen phase plate in the probe forming aperture with a virtual detector whose geometry matches that of the phase plate, a method called matched illumination detector interferometry (MIDI-STEM) [7], leads to a directly interpretable phase image and gives a linear PCTF with enhanced transfer at low spatial frequencies.

Here we introduce a 4D-STEM phase contrast imaging method (see Fig.1), ptychographic MIDI-STEM (PMIDI-STEM), which combines the advantage of a phase plate for enhanced low spatial frequency contrast transfer as demonstrated in MIDI-STEM [7], and the advantage of ptychography 4D-STEM $[3,4]$ to maximize the extraction of phase information. In this work, we perform theoretical calculations to evaluate the PCTF of PMIDI-STEM compared to that of MIDI-STEM and ptychography 4D-STEM (see Fig.1b). The phase plate significantly enhances the low spatial frequency transfer, and ptychography ensures a maximized extraction of phase information at all spatial frequencies. Experimental phase images of the Au nanoparticles on an ultrathin carbon support in Fig.1c,d) show a good contrast of the carbon support, and the diffractograms of the phase images show that PMIDISTEM results in a stronger contrast transfer compared to MIDI-STEM. Moreover, through multi-slice 
image simulations of bio-molecules at low dose conditions, we show that PMIDI-STEM is potentially capable of imaging a wide range of beam sensitive crystalline and non-crystalline materials at a low dose. [8]

[1] R. Danev, et al., Proc. Nat. Acad. Sci. 111 (2014) 15635.

[2] Knut Müller, et al., Nature Comms, 5 (2014) 5653.

[3] T.J. Pennycook, et al., Ultramicroscopy 151 (2015) 160.

[4] H. Yang, et al., Ultramicroscopy 151 (2015) 232.

[5] R. Danev, et al., Journal of biological physics, 28 (2002) 627.

[6] H. Minoda, et al., Microscopy (2015) dfv011.

[7] C Ophus, J Ciston, J Pierce et al., article in press, Nature Comms. (2016).

[8] Work at the Molecular Foundry was supported by the Office of Science, Office of Basic Energy Sciences, of the U.S. Department of Energy under Contract No. DE-AC02-05CH11231. The phase plates used in the experimental portion of this study were fabricated and tested by Jordan Pierce, Tyler Harvey, Jordan Chess and Ben McMorran. The authors also acknowledge funding from the EPSRC through grant number EP/M010708/1.
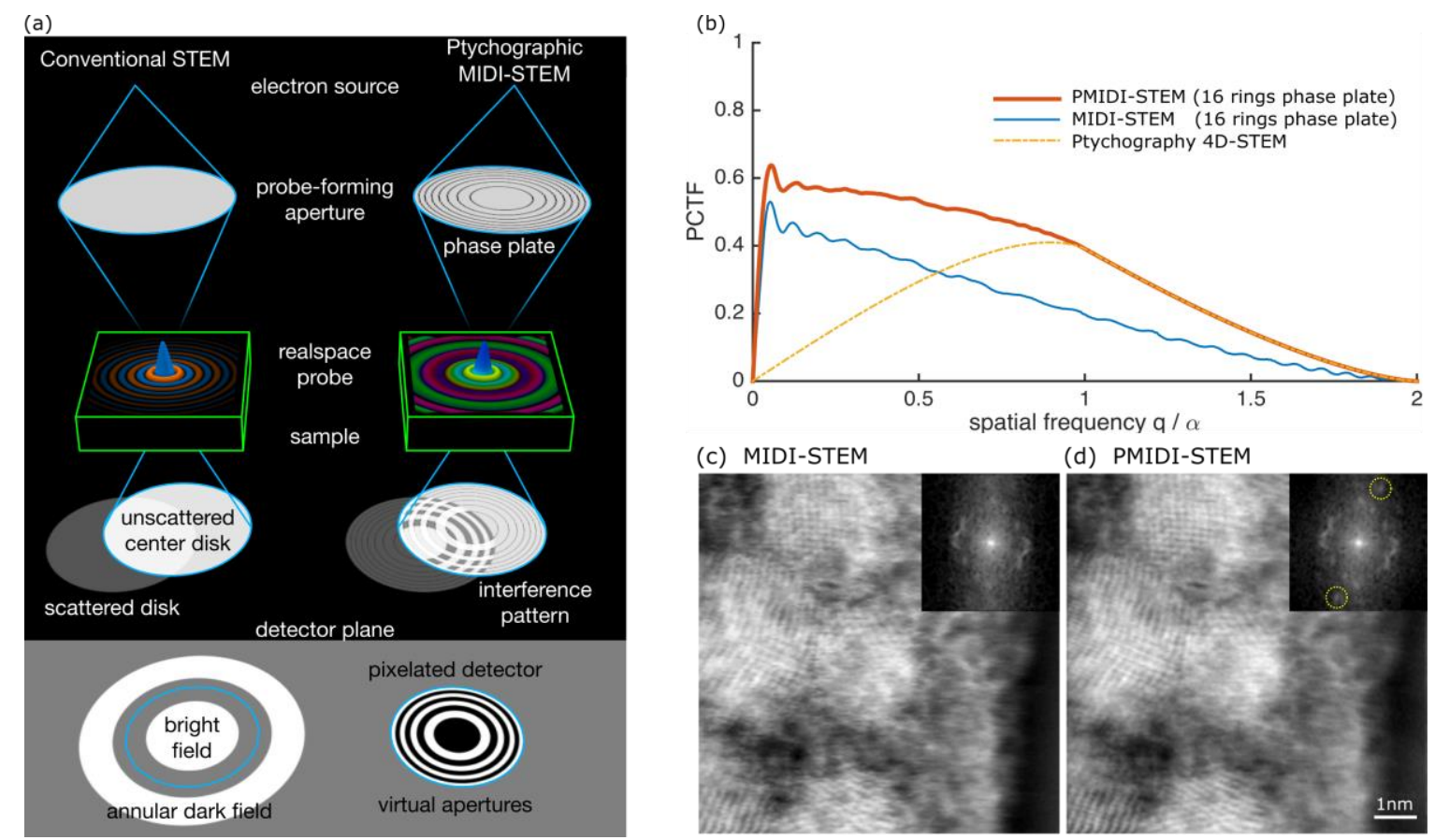

(d) PMIDI-STEM

Figure 1. (a) Schematic of the conventional STEM setup with a bright field and annular dark field detector (left), and the PMIDI-STEM setup with a pre-specimen phase plate and a pixelated detector (right). The PCTF of PMIDI-STEM, MIDI-STEM and Ptychography 4D-STEM are show in (b). (c) and (d) show the experimental results of $\mathrm{Au}$ nanoparticles on an ultrathin carbon support, using the MIDISTEM and PMIDI-STEM method, respectively. Both methods offer sensitive imaging of both the heavily scattering Au nanoparticles and weakly scattering thin carbon support, and PMIDI-STEM gives a stronger contrast transfer compared to the MIDI-STEM, as indicated from the inset diffractograms. 